\title{
Analysis of Risk Factors for Rectus Sheath Hematoma
}

\author{
Murathan Erkent ${ }^{1}$, Ramazan Topcu ${ }^{2}$, Murat Bulut Ozkan ${ }^{2}$, Murat Baki Yildirim², Orhan Aslan ${ }^{2}$ and Ismail Sezikli ${ }^{2}$ \\ ${ }^{1}$ Department of General Surgery, Medical School, Baskent University, Ankara, Turkey \\ ${ }^{2}$ Department of General Surgery, Medical School, Hitit University, Corum, Turkey
}

\begin{abstract}
Objective: To evaluate the risk factors for developing rectus sheath hematoma (RSH).

Study Design: An observational study.

Place and Duration of Study: Department of General Surgery, Hitit University School of Medicine, Erol Olcok Training and Research Hospital, Turkey; from January 2018 to April 2020.

Methodology: Patients with RSH were studied. Those with other pathologies in rectus sheath, and repeat studies, were excluded. Demographic data, presenting symptoms, comorbidities, medications administered containing anticoagulant drugs, imaging results, laboratory findings, coagulation parameters, length of hospital stay, treatments administered, type of RSH, morbidity, mortality and risk factors of increased bleeding diathesis, were recorded.

Results: Of the 61 studied patients, $56(91.8 \%)$ had at least one chronic disease, and $77 \%$ were receiving anticoagulation therapy. RSH size was significantly larger for patients taking acetylsalicylic acid than for patients taking other anticoagulants, and an RSH area less than $1,924 \mathrm{~mm}^{2}$ was associated with increased length of hospital stay. Binary logistic regression analysis showed that a unit increase in gender was associated with a 1.5-fold increase in the risk of greater RSH size, and that female gender was associated with a 45.3-fold risk of increase in the risk of RSH. Notably, if up to 4 units of erythrocyte suspension replacement is not applied for conservative treatment of RSH, RSH size may increase by 23.5 times.

Conclusion: Risk factors of RSH include chronic obstructive pulmonary disease, congestive heart failure, coronary artery disease, atrial fibrillation, asthma, hypertension, diabetes mellitus, chronic renal failure, prior abdominal surgery, female sex, older age, anticoagulant drug use and cancer-related immunosuppression.
\end{abstract}

Key Words: Rectus sheath hematoma, Conservative treatment, Anticoagulant treatment.

How to cite this article: Erkent M, Topcu R, Ozkan MB, Yildirim MB, Aslan O, Sezikli I. Analysis of Risk Factors for Rectus Sheath Hematoma. J Coll Physicians Surg Pak 2021; 31(06):633-637.

\section{INTRODUCTION}

Rectus sheath hematoma (RSH) is a cause of acute abdomen. Its frequency among patients has increased in recent years due to increased use of anticoagulants. ${ }^{1,2} \mathrm{RSH}$ is often caused by injury to the epigastric artery and its branches. ${ }^{3}$ In general, it is difficult to rule out other causes of acute abdomen to diagnose $\mathrm{RSH}^{4}{ }^{4}$ The mortality rate of RSH is around $4 \% ;{ }^{4}$ mortality in patients taking anticoagulants is higher but also preventable with early diagnosis.

The known risk factors of RSH include anticoagulant use; advanced age; female gender; pregnancy; trauma; iatrogenic injury; prior surgery; hypertension; diabetes mellitus; chronic diseases; hematological diseases; immunosuppression; and diseases that cause coughing, such as chronic obstructive pulmonary disease; and asthma. ${ }^{5}$

Correspondence to: Dr. Murathan Erkent, Department of General Surgery, Medical School, Baskent University, Ankara, Turkey

E-mail: erkentmurathan@gmail.com

Received: February 13, 2021; Revised: March 22, 2021;

Accepted: April 28, 2021

DOI: https://doi.org/10.29271/jcpsp.2021.06.633
In the differential diagnosis of acute abdomen, evaluation of patients with risk factors of RSH may prevent erroneous surgical decisions. Case reports make up the majority of the published literature on $\mathrm{RSH}^{6,7}$ and studies on the risk factors of RSH are limited. ${ }^{5,8,9}$

As the number of cases of RSH are increasing in Turkey, the objective of this study was to analyse the risk factors of RSH.

\section{METHODOLOGY}

This observational study was conducted retrospectively after obtaining approval from the Ethics Committee (No. 250, dated May 21, 2020). Computed tomography (CT) imaging reports in the Erol Olcok Training and Research Hospital computer automation system, filed between January 1, 2018, and March 4, 2020 , were screened using the keywords rectus, rectus muscle, sheath hematoma and hematoma, regardless of age or gender. This yielded 387 reports. One hundred reports containing the keyword rectus muscle and belonging to patients with diagnoses related to orbital lesions, were excluded from this study, as were 100 reports related to rectus femoris muscle, and 119 repeat reports of the same patients. Of the remaining 68 patients, 7 who were diagnosed with RSH but had abscesses during treatment, were also excluded from the study. The 
remaining 61 patients were included in this study. The demographic data, presenting symptoms, comorbidities, medications administered containing anticoagulant and antiplatelet drugs, imaging results, laboratory findings, coagulation parameters, length of hospital stay, length of follow-up period, treatments administered and type of RSH, were recorded according to Berná et al. ${ }^{10}$ Type I hematoma is unilateral and occurs within the rectus muscle; Type II hematoma may be unilateral or bilateral, within the rectus muscle or between the muscle and transversalis fascia; and Type III hematoma extends into the peritoneum and the prevesical space (Figure 1). Morbidity, mortality, and risk factors of increased bleeding diathesis were recorded for the 61 patients included in this study.

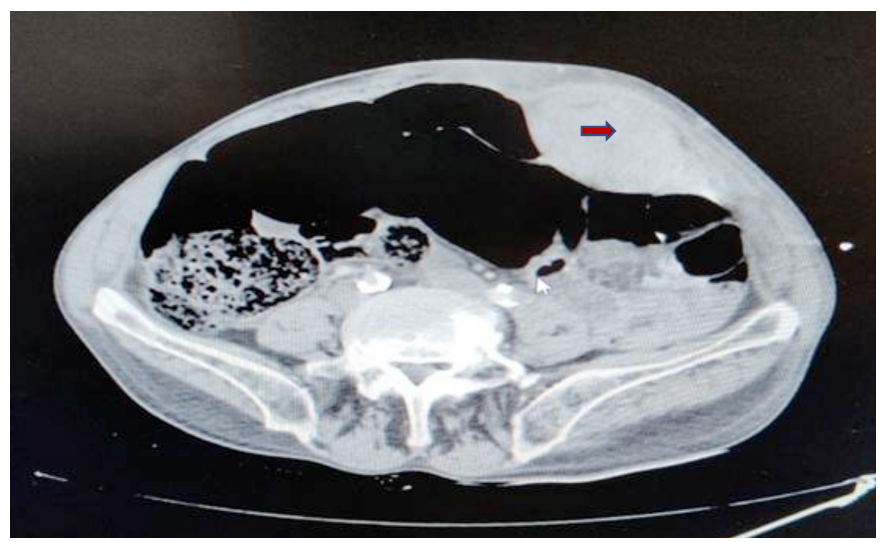

Figure 1: Type 1RSH was shown.

IBM SPSS ${ }^{\circledR}$ Statistics for Windows, version 23 , was used to analyse the study results. Descriptive statistics were expressed as numbers and percentages for categorical variables, as mean \pm standard deviation for quantitative variables, if the data were suitable for normal distribution; and as median (IQR: 25th-75th percentile), if the data were unsuitable for normal distribution. The normality distribution of the data was evaluated using the Shapiro-Wilk test. In comparing quantitative measurements according to sociodemographic characteristics and study groups, the Mann-Whitney U-test was used for two independent groups, for data unsuitable for normal distribution. A comparison of proportions according to study groups, and evaluation of correlations, was evaluated using either a Chi-square or a Fisher's Exact test. Receiver operating characteristic (ROC) curve analysis was performed to determine a cutoff value of RSH area for the different types of RSH. Binary logistic regression analysis was performed to evaluate risk factors that may result in RSH sizes greater than the cutoff value. A $p$-value $<0.05$ was considered statistically significant.

\section{RESULTS}

The demographic data and clinical features of the 61 patients included in the study are presented in Tables I and II, respectively. Fifty-six patients (91.8\%) had at least one chronic disease, and 7 patients (11.5\%) had a history of trauma. The drug use of the patients is shown in Table III. Statistically, patients on ASA had significantly higher RSH sizes than patients on other anticoagulants ( $p=0.019$; Table III). Twenty-three patients $(37.7 \%)$ were treated in outpatient settings, and 38 patients $(62.3 \%)$ were treated in inpatient settings. Fifty-nine patients (96.7\%) were followed up non-operatively, and two patients (3.3\%) underwent surgery.

Table l: Clinical and demographic characteristics of the patients.

\begin{tabular}{|l|l|}
\hline $\begin{array}{l}\text { Sex } \\
(\mathbf{n}=\mathbf{6 1 )}\end{array}$ & \\
\hline Female & $33(54.1 \%)$ \\
Male & $28(45.9 \%)$ \\
Age [years] & $64.7 \pm 17.4(25-90)$ \\
Type of RSH & \\
Type 1 & $47(77 \%)$ \\
Type 2 & $5(8.2 \%)$ \\
Type 3 & $9(14.8 \%)$ \\
& Mean + Std. Deviation \\
Hg & $11.1 \pm 2.8(\mathrm{~g} / \mathrm{dL})$ \\
Hct & $33.7 \pm 7.8$ \\
PIt & $234.7 \pm 96.8\left(\mathrm{k} / \mathrm{mm}^{3}\right)$ \\
aPTT & $27.2(18.3-67.4)$ \\
PT & $14.3(10.1-57.5)$ \\
INR & $1.22(0.91-5.8)($ IU) $)$ \\
HA & $1924(32-16060)\left(\mathrm{mm}^{2}\right)$ \\
LOS & $10.74 \pm 22.5(0-145)($ day $)$ \\
\hline RSH $=$ Rectus sheath hematoma, Hg $=$ Hemoglobin, Hct $=$ Hemotocrit, PIt $=$ \\
Platelets, aPTT = Activated partial thromboplastin time, PT = Prothrombin \\
time, INR = International normalised ratio, HA = Hematoma area, LOS = \\
length of stay.
\end{tabular}

RSH typing was performed according to CT findings. Forty-seven patients (77\%) were identified as Type 1 , five (8.2\%) as Type 2 , and nine (14.8\%) as Type 3. One patient with Type 2 and four patients with Type 3 died. The patients with Type 1 and Type 2 underwent non-operative follow-up and supportive medical therapy whereas two patients with Type 3 underwent surgery and supportive medical therapy and later died in the postoperative period. A statistically significant difference in mortality was found between the RSH types $(p<.001)$.

Regarding supportive medical therapy, 49 patients (80.3\%) were administered 1-4 units (U) of erythrocyte suspension (ES) replacement, seven patients ( $11.5 \%$ ) were administered 5-8 U, three patients (4.9\%) were administered 9-12 $\mathrm{U}$, and two patients (3.3\%) were administered $\geq 13 \mathrm{U}$. All patients also received fresh frozen plasma (FFP) and vitamin $\mathrm{K}$ (vit-K) support; 55 patients $(90.2 \%)$ were administered $1-4 \mathrm{U}$ of FFP, three patients (4.9\%) were administered 5-8 $\mathrm{U}$, two patients (3.3\%) were administered $9-12 \mathrm{U}$, and one patient (1.6\%) was administered $\geq 13 \mathrm{U}$.

RSH size ranged from $32 \mathrm{~mm}^{2}$ to $16,060 \mathrm{~mm}^{2}$. The mean length of hospital stay was $10.74 \pm 22.5$ (0-145) days. RSH was divided into two main groups - Type 1, and Types 2 and 3 - and ROC curve analysis revealed that RSH area was distinctive regarding increase in size $(p=0.001)$. The area under the ROC curve (AUC) was 0.766 , indicating a high level of distinctiveness. The $95 \%$ confidence interval (Cl) for AUC was [0.631-0.870]. Accordingly, the cutoff value distinguishing Type 1 from Types 2 and 3 was $1,924 \mathrm{~mm}^{2}$, with a sensitivity of $92.86 \%(95 \% \mathrm{Cl}$ [66.1-98.8]) and a specificity of $57.5 \%$ (95\% Cl [40.9-72.9]). Based on the sensitivity and specificity values, this cutoff value is excellent for diagnosing larger RSHs (Types 2 and 3; 92.86\%) butnot for diagnosing smaller RSHs (Type $1 ; 57.50 \%$ ). 


\section{Table II: Risk factors of RSH.}

\begin{tabular}{|c|c|c|c|c|}
\hline & & Trauma (-) & Trauma (+) & p-value \\
\hline \multirow{2}{*}{ Chronic disease } & $(-)$ & $5(9.3 \%)$ & 0 & \multirow{2}{*}{$>999$} \\
\hline & $(+)$ & $49(90.7 \%)$ & $7(100 \%)$ & \\
\hline \multirow{2}{*}{ COPD } & $(-)$ & $43(79.6 \%)$ & $7(100 \%)$ & \multirow{2}{*}{.332} \\
\hline & $(+)$ & $11(20.4 \%)$ & 0 & \\
\hline \multirow{2}{*}{$\mathrm{CHF}$} & $(-)$ & $42(77.8 \%)$ & $7(100 \%)$ & \multirow{2}{*}{.327} \\
\hline & $(+)$ & $12(22.2 \%)$ & 0 & \\
\hline \multirow{2}{*}{ CAD } & $(-)$ & $44(81.5 \%)$ & $7(100 \%)$ & \multirow{2}{*}{.587} \\
\hline & $(+)$ & $10(18.5 \%)$ & 0 & \\
\hline \multirow{2}{*}{$\mathrm{AF}$} & $(-)$ & $43(79.6 \%)$ & $6(85.7 \%)$ & \multirow{2}{*}{$>.999$} \\
\hline & $(+)$ & $11(20.4 \%)$ & $1(14.3 \%)$ & \\
\hline \multirow{2}{*}{ Asthma } & $(-)$ & $47(87.0 \%)$ & $6(85.7 \%)$ & \multirow{2}{*}{$>.999$} \\
\hline & $(+)$ & $7(13.0 \%)$ & $1(14.3 \%)$ & \\
\hline \multirow{2}{*}{ HT } & $(-)$ & $26(48.1 \%)$ & $6(85.7 \%)$ & \multirow{2}{*}{.106} \\
\hline & $(+)$ & $28(51.9 \%)$ & $1(14.3 \%)$ & \\
\hline \multirow{2}{*}{ DM } & $(-)$ & 45 (83.3\%) & $7(100 \%)$ & \multirow{2}{*}{.580} \\
\hline & $(+)$ & $9(16.7 \%)$ & 0 & \\
\hline \multirow{2}{*}{ CRF } & $(-)$ & $45(83.3 \%)$ & $7(100 \%)$ & \multirow{2}{*}{.580} \\
\hline & $(+)$ & $9(16.7 \%)$ & 0 & \\
\hline \multirow{2}{*}{ CVO } & $(-)$ & $50(92.6 \%)$ & $6(85.7 \%)$ & \multirow{2}{*}{.468} \\
\hline & $(+)$ & $4(7.4 \%)$ & $1(14.3 \%)$ & \\
\hline \multirow{2}{*}{ HVR } & $(-)$ & $49(90.7 \%)$ & $7(100 \%)$ & \multirow{2}{*}{$>.999$} \\
\hline & $(+)$ & $5(9.3 \%)$ & 0 & \\
\hline \multirow{2}{*}{ MM } & $(-)$ & $53(98.1 \%)$ & $7(100 \%)$ & \multirow{2}{*}{$>.999$} \\
\hline & $(+)$ & $1(1.9 \%)$ & 0 & \\
\hline \multirow{2}{*}{ Alzheimer } & $(-)$ & $53(98.1 \%)$ & $7(100 \%)$ & $>.999$ \\
\hline & $(+)$ & $1(1.9 \%)$ & 0 & \\
\hline \multirow{2}{*}{ Hep B } & $(-)$ & $53(98.1 \%)$ & $7(100 \%)$ & $>.999$ \\
\hline & $(+)$ & $1(1.9 \%)$ & 0 & \\
\hline \multirow{2}{*}{ OC } & $(-)$ & $53(98.1 \%)$ & $7(100 \%)$ & $>.999$ \\
\hline & $(+)$ & $1(1.9 \%)$ & 0 & \\
\hline חי & $(-)$ & $53(98.1 \%)$ & $7(100 \%)$ & $>.999$ \\
\hline PD & $(+)$ & $1(1.9 \%)$ & 0 & \\
\hline PC & $(-)$ & $53(98.1 \%)$ & $7(100 \%)$ & רم \\
\hline$B C$ & $(+)$ & $1(1.9 \%)$ & 0 & $>.999$ \\
\hline $\mathrm{PC}$ & $(-)$ & $53(98.1 \%)$ & $7(100 \%)$ & $>090$ \\
\hline$r$ & $(+)$ & $1(1.9 \%)$ & 0 & $>.999$ \\
\hline Preanancy & $(-)$ & $52(96.3 \%)$ & $7(100 \%)$ & $>999$ \\
\hline & $(+)$ & $2(3.7 \%)$ & 0 & $>.999$ \\
\hline
\end{tabular}

There was a statistically significant difference $(p<0.001)$ in the length of hospital stay between patients with RSH areas above (8; IQR = 3.1-19.8) and below (0; IQR =0-7) the cutoff value; the duration of treatment and the length of hospital stay were significantly higher in patients with $\mathrm{RSH}$ area above the cutoff value (i.e., patients with Types 2 and 3). In addition, it was assumed that prior surgery increases RSH size, and that prior vascular surgery significantly affects RSH size; however, it was later shown that prior cardiac surgery, abdominal vascular surgery, major laparotomy and minor laparotomy have no statistically significant effect on RSH size $(p>.05)$. the risk factors of Type 1 RSH and Types 2 and 3 RSH, using parameters reported in the literature as effective. Thus, variables such as drug use, age, gender, comorbidities, pregnancy status, levels of ES and FFP replacements, amount of vit-K support, hemoglobin level, hematocrit, international normalised ratio, platelet count and RSH area at the time of diagnosis were applied to the model. When insignificant variables were retrospectively removed from the regression model, it was observed that the variables age, gender, and number of ES replacements remained significant, and the determination rate of the model was $70.1 \%$. 
Table III: Patient characteristics based on anticoagulation drug usage.

\begin{tabular}{|c|c|c|c|c|c|c|c|c|}
\hline & \multicolumn{6}{|c|}{ Type of RSH } & \multirow{3}{*}{$\begin{array}{c}\text { p-value } \\
.173\end{array}$} \\
\hline & & \multicolumn{2}{|c|}{ Type 1} & \multicolumn{2}{|c|}{ Type 2} & \multicolumn{2}{|c|}{ Type 3} & \\
\hline \multirow{2}{*}{ Warfarin } & $(-)$ & 35 & $81.4 \%$ & 4 & $9.3 \%$ & 4 & $9.3 \%$ & \\
\hline & $(+)$ & 12 & $66.7 \%$ & 1 & $5.6 \%$ & 5 & $27.8 \%$ & \\
\hline \multirow{2}{*}{ LMWH } & $(-)$ & 46 & $76.7 \%$ & 5 & $8.3 \%$ & 9 & $15.0 \%$ & .859 \\
\hline & $(+)$ & 1 & $100 \%$ & 0 & $0 \%$ & 0 & $0 \%$ & \\
\hline \multirow{2}{*}{ ASA } & $(-)$ & 37 & $84.1 \%$ & 1 & $2.3 \%$ & 6 & $13.6 \%$ & .019 \\
\hline & $(+)$ & 10 & $58.8 \%$ & 4 & $23.5 \%$ & 3 & $17.6 \%$ & \\
\hline \multirow{2}{*}{ P2Y12 inhibitors } & $(-)$ & 42 & $77.8 \%$ & 3 & $5.6 \%$ & 9 & $16.7 \%$ & .074 \\
\hline & $(+)$ & 5 & $71.4 \%$ & 2 & $28.6 \%$ & 0 & $0 \%$ & \\
\hline \multirow{2}{*}{ Factor Xa inhibitors } & $(-)$ & 43 & $78.2 \%$ & 4 & $7.3 \%$ & 8 & $14.5 \%$ & .708 \\
\hline & $(+)$ & 4 & $66.7 \%$ & 1 & $16.7 \%$ & 1 & $16.7 \%$ & \\
\hline
\end{tabular}

A unit increase in age was associated with a 1.5 -fold increase in the risk of increased RSH size (odds ratio $[O R]=$ $1.514 ; 95 \% \mathrm{Cl}$ [1.100-2.083]; $p=.011$ ). Female gender was associated with a 45.3 -fold risk of increase in RSH $(O R=$ 45.319; $95 \% \mathrm{Cl}$ [1.425-1441.653]; $p=.031$ ). Therefore, male gender was a protective factor against RSH. In addition, in the treatment of RSH, if at least $4 \mathrm{U}$ of ES replacement was not applied, RSH size could increase 23.5 times $(O R=23.587 ; 95 \% \mathrm{Cl}[2.104-264.450] ; p=.010)$.

\section{DISCUSSION}

Incidence of RSH has been increasing in recent years due to increased use of anticoagulants. ${ }^{11,12}$ Therefore, in this study, the authors evaluated the current diagnostic and treatment processes of RSH as well as the risk factors of the disease, and important findings were made: First, this study statistically showed that an RSH area of $>1,924 \mathrm{~mm}^{2}$ is associated with an increase in length of hospital stay. Second, it was showed that ES replacement as conservative treatment of RSH can prevent disease progression. And third, it was found the probability of RSH and its progression increasing with the use of ASA to be significant, consistent with the literature. ${ }^{13,15}$

In the literature, some studies show the relationship between direct RSH size and failure of conservative treatment, and consensus has not emerged. ${ }^{11}$ It was found that, as RSH area increases, the duration of hospitalisation increases, and the authors think that conservative management should, therefore, be more aggressive. It was speculated that the larger the hematoma size, the more difficult it is to control bleeding, and bleeding will increase morbidity and mortality.

In the past, surgery was a widely recommended treatment for RSH; however, conservative treatment has since taken precedence. ${ }^{13}$ Indeed, in this study, 59 patients (96.7\%) were treated non-surgically, and only two patients (3.3\%) underwent surgery. Due to lack of technical resources, however, the authors could not perform embolisation using minimally invasive techniques of interventional radiology to treat patients with RSH. As conservative treatment, 1-4 U of ES replacements were administered to most patients
(80.3\%), and FFP replacement and vit-K support were also given. Most of the patients in this study had Type $1 \mathrm{RSH}$, and the rest had Types 2 and 3. Regression analysis revealed that administration of $\geq 4 \mathrm{U}$ of ES replacement can prevent RSH progression by 23.5 times. In the literature on conservative treatment, there are no reports of similar results when ES replacement is used as supportive therapy.

In patients with non-traumatic RSH, the most common comorbidities were related to the cardiovascular system. The increase in the incidence of RSH, then, may be due to the need for anticoagulants as treatment for cardiovascular disease.

Similar to reports in the literature, in this study, RSH was more common in women $(54.1 \%)$ than in men. In fact, female gender was associated with a 45.3-fold increase in the risk of RSH. Thus, male gender may be a protective factor against RSH. As reported in other studies, this may be due to the anatomical differences in the rectus muscle between the sexes, explaining the weaker abdominal wall muscles in women, and due to increased abdominal distension during pregnancy, as two patients in our study were pregnant. ${ }^{14}$ Similarly, a unit increase in age was associated with a 1.5-fold increase in the risk of RSH size.

In this study, two out of four patients with Type 3, and one patient with Type 2, died prior to surgery, and the other two patients with Type 3 died in the postoperative period. The mortality rate in our study was higher than that reported in other studies. ${ }^{15,16}$ This could be because the patients scheduled for surgery in our study could not undergo embolisation, due to a lack of technical resources in this hospital.

As reported in earlier studies, prior surgery may be associated with an increase in RSH size. However, in the present study, the effect of prior cardiac surgery, abdominal vascular surgery, major laparotomy or minor laparotomy on RSH size was not statistically significant.

The major limitation of this study was its retrospective design. In addition, the authors could not reach BMI (body mass index) values that might be important in this data. 
Patients with RSH were included in the study, and risk factors were investigated, but a comparison to a patient group without RSH but with similar comorbidities as the patients with RSH was not made. This may explain the statistical insignificance found with some of the risk factors of RSH.

\section{CONCLUSION}

Increased use of anticoagulants is a major risk factor of RSH. Other risk factors include old age, female gender, chronic obstructive pulmonary disease, congestive heart failure, coronary artery disease, atrial fibrillation, asthma, hypertension, diabetes mellitus, chronic renal failure, prior abdominal surgery and cancer-related immunosuppression. The authors recommend ES replacement in patients with $\mathrm{RSH}$ sizes larger than 1,924 $\mathrm{mm}^{2}$, and using ASA as an anticoagulant. There is a need for multicenter studies with larger population samples to assess the risk factors of RSH in more detail.

\section{ETHICAL APPROVAL:}

This study was approved by the Ethical Review Committee of the Hitit University School of Medicine.

\section{PATIENTS' CONSENT:}

Informed consents were obtained from all patients.

\section{CONFLICT OF INTEREST:}

The authors declared no conflict of interest.

\section{AUTHORS' CONTRIBUTION:}

ME: Study design, data collection, statistical analysis, data Interpretation, manuscript preparation, literature search, funds collection.

RT: Study design, data collection, manuscript preparation, literature search.

MBO, MBY, OA: Study design, data collection, manuscript preparation, funds collection.

IS: Data collection, funds collection, literature search.

\section{REFERENCES}

1. Titone C, Lipsius M, Krakauer JS. 'Spontaneous' hematoma of the rectus abdominis muscle: Critical review of 50 cases with emphasis on early diagnosis and treatment. Surgery 1972; 72(4):568-72.

2. Warren $M$ H, Bhattacharya B, Maung AA, Davis KA. Contemporary management of spontaneous retroperitoneal and rectus sheath hematomas: Management of spontaneous hematomas. In: Am J Surg 2020; 219(4):707-10. doi: 10.1016/j.amjsurg.2019.05.002.

3. Torcia P, Rossi UG, Squarza S, Cariati M. Spontaneous hematoma of the rectus sheath: Urgent embolisation with squidperi liquid embolic device. Case Rep Radiol 2017; 2017:3420313. doi: 10.1155/2017/3420313.

4. Hatjipetrou A, Anyfantakis D, Kastanakis M. Rectus sheath hematoma: A review of the literature. Int J Surg 2015; 13:267-271. doi: 10.1016/j.ijsu.2014.12.015.

5. Sheth H, DiNella J, Janov C, Smith R, Kaldas H. Evaluation of Rectus Sheath Hematoma (RSH). Chest 2012; 142:841A.

6. Bello G, Blanco P. Giant rectus sheath hematoma. Ultrasound J 2019; 11(1):13. doi: 10.1186/s13089-019-0129-4.

7. Azharuddin M, Gupta M, Maniar M. Subcutaneous heparin leads to rectus sheath hematoma: A rare complication. Cureus 2018; 10(6):e2769. doi: 10.7759/cureus.2769.

8. Cherry WB, Mueller PS. Rectus sheath hematoma: Review of 126 cases at a single institution. Medicine (Baltimore) 2006; 85(2):105-110. doi: 10.1097/01.md.0000216818.13067.5a.

9. Aktürk OM, Kayılıoğlu SI, Aydoğan I, Dinç T, Dinç T, Yildiz B, et al. Spontaneous rectus sheath hematoma: An overview of 4-Year single center experience. Indian J Surg 2015; 77(Suppl 3):1219-21. doi: 10.1007/s12262-015-1257-0.

10. Berná JD, Garcia-Medina V, Guirao J, Garcia-Medina J. Rectus sheath hematoma: Diagnostic classification by CT. Abdom Imaging 1996; 21(1):62-64. doi: 10.1007/s002619900011.

11. Ibrahim M, Enambir J, Zhang M. Spontaneous rectus sheath hematoma complicating therapeutic heparinisation. Am J Ther 2018; 25(6):e704-5. doi: 10.1097/MJT.00000000 00000719.

12. Çarkman S, Özben V, Zengin K, Somuncu E, Karatas A. Spontaneous rectus sheath hematoma: An analysis of 15 cases. Ulus Travma Acil Cerrahi Derg 2010; 16(6):532-6.

13. Contrella BN, Park AW, Wilkins LR, Sheeran D, Hassinger TE, Angle JF. Spontaneous rectus sheath hematoma: Factors predictive of conservative management failure. J Vasc Interv Radiol 2020; 31(2):323-30. doi: 10.1016/j.jvir.2019.06.009.

14. Dağ A, Özcan T, Türkmenoğlu Ö, Çolak T, Karaca K, Canbaz $\mathrm{H}$, et al. Spontaneous rectus sheath hematoma in patients on anticoagulation therapy. Ulus Travma Acil Cerrahi Derg 2011; 17(3):210-14. doi: 10.5505/tjtes.2011.84669.

15. Smithson A, Ruiz J, Perello R, Valverde M, Ramos J, Garzo L. Diagnostic and management of spontaneous rectus sheath hematoma. Eur J Intern Med 2013; 24(6):579-82. doi: 10.1016/j.ejim.2013.02.016.

16. Isik A, Peker K, Soyturk M, Firat D, Yoruker U, Yilmaz I. Diagnostic evaluation and treatment of patients with rectus abdominis hematoma. Cir Esp 2015; 93(9):580-8. doi: 10.1016/j.ciresp.2015.02.014. 